$>$ Objective: The postmenstrual bleeding with cesarean-induced diverticulum is a bothersome symptom and can be confused with other abnormal-uterine bleeding disease. The aim of the present study was to evaluate the efficacy and safety of laparoscopic combined with hysteroscopic repair vs operative hysteroscopy in treatment of symptomatic cesarean-induced diverticulum.

Methods: Eighty-two women with cesarean-induced diverticulum who underwent either laparoscopic combined with hysteroscopic repair or operative hysteroscopy between January 2012 and September 2015 were retrospectively included in the study. We evaluated the menstrual duration before and after the surgery, the width, length and depth of the niche, operating time, blood loss, complications, and postoperative pregnancy status.

Results: $62.2 \%(51 / 82)$ patients started to have postmenstrual bleeding immediately when they gained regular menstruation after their cesarean section. The laparoscopic group has longer operative time and greater blood loss $(p<0.001)$. Both groups have a significant improvement in their menstruation duration ( $p<$ 0.001 ). The patients in the laparoscopic repair group had a greater improvement in bringing the uterine bleeding into normal menstruation duration $(P=0.046)$ and the same in pregnancy rate $(P=0.505)$. The interval between operation and pregnancy in laparoscopic combined with hysteroscopic group was much longer than the hysteroscopic group (27 vs 10 months, $p<0.001$ ).

Conclusion: the laparoscopic repair exhibited a better efficiency in bringing the uterine bleeding into normal menstruation duration and in improving the pregnancy rate However, there was not a clear absolute advantage for one of the two type of surgeries. Thus, patients with post-cesarean section diverticulum (PCSD) should choose with caution the type of surgery,

\section{Laparoscopic combined with hysteroscopic repair or operative hysteroscopy in the treatment of symptomatic cesarean-induced diverticulum}

Bingqing Lv, Xi Xie, Chaobin Liu, Yuan Lin

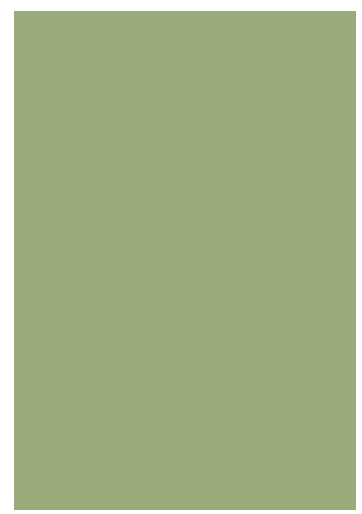

Department of Gynecology and Obstetrics, Fujian Province Maternity and Children's Hospital, affiliated hospital of Fujian Medical University, No.18 Anshan Road, Gulou District Fuzhou City, Fujian Province 350001 , PR China.

Corresponding author: Xi Xie 50123757@qq.com

considering the residual myometrial thickness (RMT), the age, the desire for further fertility and ovarian reserve capacity. < Key words: cesarean-induced diverticulum; cesarean-section defect niche; hysteroscpic surgery; postoperative pregnancy; postmenstrual bleeding.

\section{Introduction}

The number of deliveries by cesarean section has been increasing steadily worldwide in recent decades, especially in China. It has been reported that half of the deliveries in 2010 were by cesareans [1] despite the short-term risks such as an increased blood loss, the high risk of infection and the thromboprophylaxis $[2,3]$. Cesarean section carries long-term sequelae, which can adversely affect subsequent pregnancies. In a recent study, $64.5 \%$ of patients who underwent cesarean section developed uterine incision diverticulum within 6 to 12 weeks after surgery, which can cause abnormal uterine bleeding. Women with a single caesarean section had a $62 \%$ risk of developing niche, compared with $68.2 \%$ of women with two caesarean 
sections and $77.8 \%$ of women with three-times caesarean sections [4]. However, the syndrome often confuses with other uterine diseases. Some inexperienced doctors, especially from the underdeveloped communities hardly notice this defect. Currently, the treatment of this diverticulum may be performed by laparoscopic, combined laparoscopic vaginal surgery or vaginal surgery and operative hysteroscopy, transabdominal or transvaginal, all minimally invasive approaches to improve symptoms and restore fertility $[5,6]$. However, there is no guideline to indicate which technique should be selected. In the present study, the diagnosis and the prevalence of associated symptoms will be reviewed; how the various surgical approaches (hysteroscopic or laparoscopic) may improve clinical problems related to cesarean section scar will be also discussed.

\section{Material and methods}

\section{Patients}

This study was conducted at the Fujian Provincial Maternity and Children's Hospital, affiliated hospital of Fujian Medical University. We conducted a retrospective analysis of data relating to 82 patients with symptomatic post-cesarean section uterine diverticulum who underwent either combined hysteroscopic and laparoscopic repair or hysteroscpic surgery only between January 2012 and September 2015. The patients were examined within the tenth day of their menstruation by transvaginal three-dimensional (3D) color doppler ultrasound. The diagnosis of post-cesarean section uterine diverticulum and the inclusion criteria are based on the following: (1) at least one cesarean delivery was documented; (2) the presence of asonographically documented diverticulum; (3) the exclusion criteria included pregnant women; women using intrauterine devices; women with previous menstrual irregularities, coagulation disorders, myomas, polyps, endometrial hyperplasia, and cancers of the cervix or endometrium. The study was approved by the Ethics Committee of the Fujian Procincial Maternity and Children's Hospital, and informed written consent was obtained from all patients.

\section{Laparoscopic combined with hysteroscopic repair}

All patients underwent the surgery procedure in their follicular phase. All procedures were conducted under general anesthesia, and patients were placed in the trendelenburg (head-down) position. An electric scalpel (GebrOder Martin ME maxium) was used to incise the peritoneal fold over the bladder and pushed down the bladder below the lower edge of the diverticulum. Under hysteroscopic examination, the operating surgeon confirmed the location and size of the diverticulum. In addition, a surgical assistant used a surgical probe to locate the weakest part of the diverticulum. The surgeon then used an electric coagulation hook to open the diverticulum laparoscopically. The scar tissue was excised from the edges of the defect until the edge was trimmed and with healthy myometrium. We used then the doublelayer closure: Vicryl (Ethicon, 1-0) was used to perform continuous full thickness suturing including the endometrial layer. After the first layer suture, the hysteroscopic examination was performed to confirm that the diverticulum was completely resected. Then, a continuous mattress suturing (Vicryl, Ethicon, 1-0) the myometrium was used to strengthen the wound. After bleeding assessment, the peritoneal fold of the bladder was finally closed. The cefaclor antibiotics were used within $48 \mathrm{~h}$ after surgery.

Operative hysteroscopy was performed during anesthesia using a unipolar electrical current which was set at $40 \mathrm{~W}$ for cutting and $30 \mathrm{~W}$ for coagulation resectoscope with a 90_loop and a 0 _optic (Karl Storz GmbH \& Co.KG). The uterine cavity was distended using normal saline; an automatic pressure infusor (52-13-200ELC; VBM Medizintechnik, GmbH\& Co., Sulza.N., Germany) was used to provide positive pressure ( $21 \mathrm{kpal})$. The superior edge of the defect was resected using a cutting loop and pure cutting current, adapting the technique in order to remove the anterior scar tissue completely and to align the surface to the cervix. The electrical rollerball was used to cauterize the dilated blood vessels and to destroy the endometrium that lays on the roof of the diverticulum (Figure I). The surgery was ended when no bleeding was observed under direct visualization.

\section{Evaluation index}

The evaluation index includes the patients age, the number of cesarean deliveries, the duration of menstruation before and after surgery, the length, width and depth of the diverticulumas determined by doppler ultrasound, the operative time, the operative blood loss, the presence of any surgical complications, and the patients future fertility. All the above indexes were recorded and compared between the two groups. The clinical efficacy of surgery was classified as: greatly improved (regaining normal menstrual periods), improved (at least 3 days shorter than the preoperative menstrual periods but not normal) and non-improved (similar menstrual periods before and after surgery).

\section{Statistical analysis}

The measurement data comparison between groups was made based on the student t-test of the sample mean. The enumeration data were expressed with a relative number, and the comparison between groups was made based on the $\chi^{2}$ test. The data, not normally distributed were analyzed by a Mann-Whitney $U$ test. The Wilcoxon signed-rank test was performed to examine changes after surgery. Numbers and percentages were calculated for categorical variables and tested by the $\chi^{2}$ test.

The correlation between the length, width, depth of the diverticulum and the length of the patients menstrual periods were analyzed by the Pearson correlation 

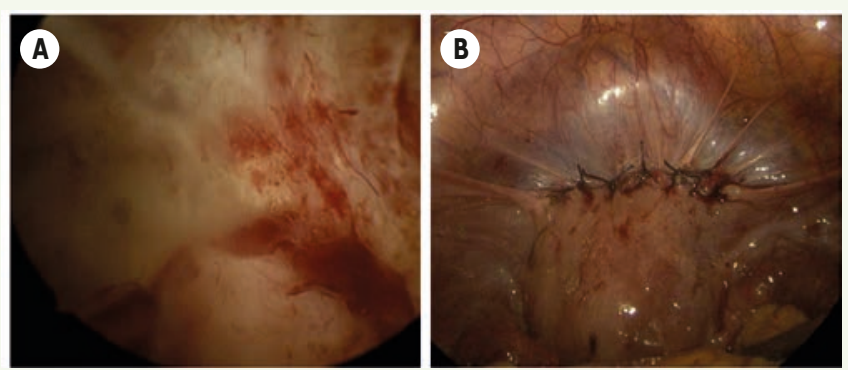

Figure 1. Laparoscopic combined with hysteroscopic repair of cesarean-induced diverticulum (A: before surgery; B: post-surgery).

\begin{tabular}{lccc}
\hline Item & $\begin{array}{c}\text { Laparoscopic } \\
\text { repair }\end{array}$ & $\begin{array}{c}\text { Hysteroscopic } \\
\text { repair }\end{array}$ & P \\
\hline Age (years) & $31.2(23-43)$ & $31.8(24-43)$ & 0.423 \\
\hline Numbers of CS & $1.2(1-3)$ & $1.2(1-2)$ & 0.758 \\
\hline $\begin{array}{l}\text { Post-CS menstrua- } \\
\text { tion duration (day) }\end{array}$ & $13(7-24)$ & $14(8-20)$ & 0.407 \\
\hline RMT (mm) & $0.9(0.1-3.9)$ & $1.0(0.5-3.8)$ & 0.088 \\
\hline $\begin{array}{l}\text { Depth of niche } \\
\text { (mm) }\end{array}$ & $10.1(6-32)$ & $8.9(2-35)$ & 0.225 \\
\hline $\begin{array}{l}\text { Length (mm) } \\
\text { Width (mm) }\end{array}$ & $8.5(3-23)$ & $7.4(2-17)$ & 0.206 \\
\hline $\begin{array}{l}\text { Blood loss (mL) } \\
\text { Operative time (h) }\end{array}$ & $18.3(3-13)$ & $6.5(2-12)$ & 0.144 \\
\hline $\begin{array}{l}\text { Menstruation } \\
\text { duration } \\
\text { after surgery }\end{array}$ & $2.0(0.5-3.2)$ & $0.8(0.2-2.0)$ & $<0.05$ \\
\hline
\end{tabular}

Table 1. Characteristics of the included patients.

test. Statistical significance was determined by a two-tailed $p$ value of $<0.05$. All statistical tests were conducted using the spss 16.0 .2 software.

\section{Results}

\section{General characteristics of the included patients}

Fifty-one patients (51/82) started having postmenstrual bleeding immediately when they gained regular menstruation after their cesarean section according to their medical documents. The laparoscopic combined with hysteroscopic group comprised 31 women, and the operative hysteroscopy group comprised 51 women. The characteristics of these 82 patients are summarized in Table 1. The groups were similar in the following baseline features: patient age, number of previous cesarean deliveries, length of preoperative menstrual periods, length, width and depth of the diverticulum, residual myometrial thickness $(P>0.05)$. The Laparoscopic combined with hysteroscopic group had longer operative times ( $2 h$ vs $0.8 h, p<0.001$ ) and greater blood loss ( 18.4 vs $5.2 \mathrm{~mL}, p<0.001$ ). The length of patient menstrual periods before surgery had no correlation with the length, width or depth of the diverticulum. (Pearson correlation test: $R_{\text {length }}=0.121, P=0.279$; $R_{\text {width }}=0.222, P=0.045 ; R_{\text {depth }}=0.121, P=0.278$ ).

\section{Follow-up results}

The follow-up period ranged from 13 to 36 months (mean, 21 months), with 30 patients in laparoscopic combined with hysteroscopic group and 47 patients in hysteroscopic group having a follow-up through email, phone call or text messages. The treatment efficacy of post-operative menstruation duration of both groups are summarized in Table 2. The median change was 6.7 days (4-10) in laparoscopic combined with hysteroscopic group $(P<0.001)$, and $7.7(4-14)$ in the operative hysteroscopy group $(p<0.001)$. The 20 non-improved patients started their postmenstrual bleeding within 3 months after surgery.

\section{Effects on pregnancy}

Thirteen patients in laparoscopic combined with hysteroscopic group desired further fertility before the surgery. Eight of them were able to achieve pregnancy $(61.5 \%)$. It included miscarriage of 1 patient after 8 weeks, 2 at their midterm of gestation, 3 at 35-38 weeks of gestation, while 2 had mature delivery by cesareansection. 11 out of 15 patients in the hysteroscopic group were able to achieve pregnancy $(73.3 \%)$, including 3 patients in early gestation, 3 in their midterm of gestation. One patient gave birth in the $34^{+2}$ weeks by vaginal delivery, and 4 had a premature delivery by CS. There was no statistical difference between the two groups in the pregnancy rate. The residual myometrial thickness (RMT) of the pregnant women in the hysteroscopic group ranged from 0.7 to $3.1 \mathrm{~mm}$. None of them was suffering from uterine rupture. The interval between operation and pregnancy in the laparoscopic combined with hysteroscopic group was 27 months (15-36) which was much longer than the hysteroscopic group [10 months $(5-20)](p<0.001)$.

\section{Discussion}

The defects in cesarean section scars are common [7]. The prevalence of caesarean scar diverticulum was found to vary between $56 \%$ and $84 \%$ according to a 


\begin{tabular}{lccc}
\hline Group & $\begin{array}{c}\text { Significantly } \\
\text { improved }\end{array}$ & Improved & Unimproved \\
$\begin{array}{l}\text { Laparoscopic } \\
\text { repair }\end{array}$ & $14(46.7)$ & $10(33.3)$ & $6(20.0)$ \\
$\begin{array}{l}\text { Hysteroscopic } \\
\text { repair }\end{array}$ & $11(23.4)$ & $22(46.8)$ & $14(29.8)$ \\
\hline
\end{tabular}

Table 2. Menstruation duration after surgery in the two groups.

published study [8]. As not all women with an history of cesarean section develop a diverticulum, it is of interest to identify the symptoms that may predict them. There is growing interest in possible associations between the presence of a niche and various gynecological symptoms, and in the mechanisms behind the development of these symptoms. Among women with cesarean scar defect, abnormal uterine bleeding, a common symptom reported to be associated with the presence of ache, was found in 28.9-82\% of cases [9]. The most typical and bothersome symptom is postmenstrual bleeding (all our cases) characterized by the discharge of a dark hematic material collected in the scar pouch during the days after menstruation. The bloody fluid retention which is due to the atypical anatomical defect and the hemorrhage from the scarred area can be the cause of this syndrome. It has been reported that one out of three women with a niche has a abnormal menstrual bleeding, compared with one out of ten women without a niche. The syndrome occurs one year after caesarean section was strongly related to the presence of a niche [4]. Our result shows that $62.2 \%(51 / 82)$ patients start having postmenstrual bleeding immediately when they gain regular menstruation after their cesarean section. The defect of cesarean scar has been reported to be as high as $24 \%$ to $64.5 \%$ in women, 6 to 12 weeks after cesarean section [ 4 , 8]. Due to breast-feeding, part of the women gain their menstruation after the cesarean section diverticulum is formed. The bloody fluid retention which is due to the atypical anatomical defect can be the cause of what was found herein. Wang C.B [10] believes that the size of the cesarean scar defect is associated with postmenstrual bleeding. van der Voet [4] found that postmenstrual spotting was related to a residual myometrium: women with residual myometrium at the site of the uterine scar measuring $<50 \%$ of the adjacent myometrial thickness had postmenstrual spotting more often than women with a residual myometrial thickness $>50 \%$ of the adjacent myometrial thickness. In our study, we have not started measuring the residual myometrium vs the adjacent myometrial thickness. However, there was no correlation between the length, width or depth of the diverticulum and the length of patient menstrual periods. The postmenstrual bleeding of cesarean section diverticulum may have multiple physiopathologic mechanisms along with an atypical anatomy that requires further randomized research.

The surgical treatment of symptomatic women with cesarean scar defect is the partial resection of scarring by hysteroscopy and complete resection of the scar tissue, the suture of the healthy tissue through abdominal or through vaginal surgery. To date, there are no guidelines for the selection of operative strategies for this defect. Some reports have cited an RMT of $<2 \mathrm{~mm}$ as a rough indicator of the risk of uterine rupture during a trial of labor after cesarean delivery [11, 12]. Furthermore, a RMT of $<2 \mathrm{~mm}$ has been shown to increase the risk of uterine rupture and bladder injury during hysteroscopic surgery [13]. Satoshi Tanimura used RMT and the position of the uterus as criteria for the selection of surgery to treat cesarean scar defect and showed that hysteroscopic surgery is only indicated for women with an RMT of $\geq 2.5 \mathrm{~mm}$ [14]. But in the present study, there are 25 RMT in the hysteroscopic group $<2 \mathrm{~mm}$ and no significant difference between the two group. None of the 82 patients got uterine rupture, bladder injury and infection. Thus, we assume that the RMT is not the essential criteria selection of surgery. The limited operative time and the surgical proficiency can help to eliminate the risk.

Due to the short operative time and the smaller loss of blood, operative hysteroscopy can be performed if the adjacent myometrium exists and is continuous. However, whether the myometrium can bear the pregnancy remains unknown. In the present study, we performed the Laparoscopic Repair when the patient RMT was $<\mathrm{lmm}$, with the intention of a future pregnancy.

The clinical efficacy of these two types of surgery varies according to different articles. It has been reported that the hysteroscopic repair of cesarean scar defect is associated with an improvement in uterine bleeding in $59 \%$ to $100 \%$ of cases and the pregnancy rate in $77.8 \%$ to $100 \%$ of cases. Improvement in uterine bleeding after vaginal repair occurs in $89 \%$ to $93.5 \%$ of cases. Laparoscopic repair leads to uterine bleeding improvement in $86 \%$ of cases, with a pregnancy rate of $86 \%$ [9]. In our finding, the laparoscopic repair was more efficient in bringing the uterine bleeding into a normal menstruation duration and similar in the pregnancy rate. In China, we recommend that women have at least a one year inter-pregnancy interval after cesarean section. We favor the same interval for women after laparoscopic repair and 3 months for the hysteroscopic repair, the laparoscopic repair having a much longer contraceptive time than the hysteroscopic repair, which may affect the pregnancy rate due to our short observation time. In our opinion, women with advanced age (>40 years) or women with lower ovarian reserve capacity are not suitable for laparoscopic repair. If a patient with an advanced age and a RMT <lmm still has plan for a further pregnancy, she should be fully informed of the consequence and choose the operation with caution. An oocyte retrieval should be done before the laparoscopic surgery to preserve the fertility. To date, it has been 
reported that postoperative menstruation and imaging data did not differ significantly between 3 months and 6 months after vaginal repair, suggesting that follow-up at 3 months represents an adequate endpoint for evaluating the effectiveness of surgery [15]. This may be an indication that there is still a space for shortening the contraceptive time for the women who underwent laparoscopic repair.

The limitations of the current study include its retrospective nature, its limited sample size. Moreover, we did not examine the nidus size after the surgery to evaluate the efficacy of the methods. A large, randomized controlled trial and a longer follow-up period are needed to verify our findings.

\section{Conclusion}

Postmenstrual bleeding occurred immediately when the patient gained regular menstruation after the cesarean section suggested the existence of a cesarean section diverticulum. As the two type of surgeries can be still envisionned, patients with PCSD should choose with caution the method to be used, considering the RMT, the age, the desire for further fertility and the ovarian reserve capacity. However, the hysteroscopic repair may be a more suitable choice in a number of patients. $\diamond$

\section{ACKNOWLEDGEMENTS}

This investigation was supported by the construction project of clinical specialty in Fujian Province (project No. 2012149).

\section{DISCLOSURE STATEMENT}

The authors have no conflict of interest to declare.

\section{REFERENCES}

1. Hellerstein S, Feldman S, Duan T. China's $50 \%$ caesarean delivery rate: is it too high. BJOG 2015;122:160-4
2. Jolly J, Walker J, Bhabra K. Subsequent obstetric performance related to primary mode of delivery. Br J Obstet Gynaecol 1999;106:227-32.

3. LaSala AP, Berkeley AS. Primary cesarean section and subsequent fertility. Am J Obstet Gynecol 1987;157:379-83.

4. van der Voet LF, de Vaate AM B, Veersema S, Brölmann HA, Huirne JA. Long-term complications of caesarean section. The niche in the scar: a prospective cohort study on niche prevalence and its relation to abnormal uterine bleeding. BJOG 2014;121:236-44.

5. Florio P, Filippeschi M, Moncini I, Marra E, Franchini M, Gubbini G. Hysteroscopic treatment of the cesarean-induced isthmocele in restoring infertility. Curr Opin Obstet Gynecol 2012;24:180-6.

6. Xie H, Wu Y, Yu F, He M, Cao M, Yao S. A comparison of vaginal surgery and operative hysteroscopy for the treatment of cesarean-induced isthmocele: a retrospective review. Gynecol Obstet Invest 2014;77:78-83.

7. Osser OV, Jokubkiene L, Valentin L. High prevalence of defects in Cesarean section scars at transvaginal ultrasound examination. Ultrasound Obstet Gynecol 2009;34:90-7.

8. de Vaate AJ B, van der Voet LF, Naji O, Witmer M, Veersema S, Brölmann HA, Bourne T, Huirne JA. Prevalence, potential risk factors for development and symptoms related to the presence of uterine niches following Cesarean section: systematic review. Ultrasound Obstet Gynecol 2014;43:372-82.

9. Tulandi T, Cohen A. Emerging Manifestations of Cesarean Scar Defect in Reproductive-aged Women. J Minim Invasive Gynecol 2016;23:893-902.

10. Wang CB, Chiu WW, Lee CY, Sun YL, Lin YH, Tseng CJ. Cesarean scar defect: correlation between Cesarean section number, defect size, clinical symptoms and uterine position. Ultrasound Obstet Gynecol 2009;34:85-9.

11. Bujold $\varepsilon$, Jastrow N, Simoneau J, Brunet S, Gauthier RJ. Prediction of complete uterine rupture by sonographic evaluation of the lower uterine segment. Am J Obstet Gynecol 2009;201:320.el-6.

12. Kok N, Wiersma IC, Opmeer BC, de Graaf IM, Mol BW, Pajkrt E. Sonographic measurement of lower uterine segment thickness to predict uterine rupture during a trial of labor in women with previous Cesarean section: a metaanalysis. Ultrasound Obstet Gynecol 2013;42:132-9.

13. Chang Y, Tsai $\varepsilon M$, Long CY, Lee CL, Kay N. Resectoscopic treatment combined with sonohysterographic evaluation of women with postmenstrual bleeding as a result of previous cesarean delivery scar defects. Am J Obstet Gynecol 2009;200:370.el-4.

14. Tanimura S, Funamoto H, Hosono T, Shitano Y, Nakashima M, Ametani $Y$, Nakano T. New diagnostic criteria and operative strategy for cesarean scar syndrome: Endoscopic repair for secondary infertility caused by cesarean scar defect. J Obstet Gynaecol Res 2015;41:1363-9.

15. Zhou J, Yao M, Wang H, Tan W, Chen P, Wang X. Vaginal Repair of Cesarean Section Scar Diverticula that Resulted in Improved Postoperative Menstruation. J Minim Invasive Gynecol 2016;23:969-78. 